Article

\title{
A New Scheme Using Cubic B-Spline to Solve Non-Linear Differential Equations Arising in Visco-Elastic Flows and Hydrodynamic Stability Problems
}

\author{
Asifa Tassaddiq ${ }^{1}\left(\mathbb{D}\right.$, Aasma Khalid ${ }^{2,3}$, Muhammad Nawaz Naeem ${ }^{3}$, Abdul Ghaffar ${ }^{4}$ (D), \\ Faheem Khan ${ }^{5}$, Samsul Ariffin Abdul Karim ${ }^{6}$ and Kottakkaran Sooppy Nisar ${ }^{7, *}$ (D) \\ 1 College of Computer and Information Sciences, Majmaah University, Al-Majmaah 11952, Saudi Arabia; \\ a.tassaddiq@mu.edu.sa \\ 2 Department of Mathematics, Government College Women University Faisalabad, Faisalabad 38023, \\ Pakistan; aasmakhalid@gcwuf.edu.pk \\ 3 Department of Mathematics, Government College University Faisalabad, Faisalabad 38023, Pakistan; \\ mnawaznaeem@yahoo.com \\ 4 Department of Mathematical Sciences, BUITEMS, Quetta 87300, Pakistan; abdulghaffar.jaffar@gmail.com \\ 5 Department of Mathematics, University of Sargodha, Sargodha 40100, Pakistan; fahimscholar@gmail.com \\ 6 Fundamental and Applied Sciences Department and Centre for Smart Grid Energy Research (CSMER), \\ Institute of Autonomous System, Universiti Teknologi PETRONAS, Bandar Seri Iskandar, Seri Iskandar \\ 32610, Perak Darul Ridzuan, Malaysia; samsul_ariffin@utp.edu.my \\ 7 Department of Mathematics, College of Arts and Sciences, Prince Sattam bin Abdulaziz University, Wadi \\ Aldawaser 11991, Saudi Arabia \\ * Correspondence: n.sooppy@psau.edu.sa; Tel.: +966-563-456-976
}

Received: 4 August 2019; Accepted: 28 October 2019; Published: 8 November 2019

\begin{abstract}
This study deals with the numerical solution of the non-linear differential equations (DEs) arising in the study of hydrodynamics and hydro-magnetic stability problems using a new cubic B-spline scheme (CBS). The main idea is that we have modified the boundary value problems (BVPs) to produce a new system of linear equations. The algorithm developed here is not only for the approximation solutions of the $10^{\text {th }}$ order BVPs but also estimate from 1st derivative to $10^{\text {th }}$ derivative of the exact solution as well. Some examples are illustrated to show the feasibility and competence of the proposed scheme.
\end{abstract}

Keywords: non-linear differential equation; cubic B-spline; central finite difference approximations; absolute errors

MSC: 34K10; 34K28; 42A10; 65D05; 65D07

\section{Introduction}

Recent research in the field of hydrodynamic and hydromagnetics stability have found the presence of a family of problems in differential equations (DEs) of a high order, and which have real mathematical interest. There are various approximate (numerical) methods in the literature that have been used for the solution of boundary value problems (BVPs). The existence and uniqueness to finding the solution of higher order BVPs are systematically examined in [1]. The BVPs of higher order DEs have been examined due to their significance and the potential for applications in applied sciences. To find the analytical solutions of such BVPs analytically is very tough and are available in very few cases. Very few researchers have tried the numerical solution of $10^{\text {th }}$ order BVPs. Some of the approximate techniques have been established over the years to the numerical solution for these kinds 
of BVPs. In [2,3], the authors has solved $10^{\text {th }}$ and $12^{\text {th }}$ order BVPs using the Adomian decomposition method (ADM) involving Green's function. The homotopy perturbation approach was utilized in [4] to solve BVPs of $10^{\text {th }}$ order. When a uniform magnetic field is applied across the fluid in the direction of gravity, the instability sets now as ordinary convection and it is modeled by $10^{\text {th }}$ order BVPs as discussed in [5]. In [6], established approximate techniques for solving the $10^{\text {th }}$ order non-linear BVPs occurring in thermal instability.

Numerical methods for the solution of non-linear BVPs of order $2 \mathrm{~m}$ were found in [7]. An effective numerical procedure DTM for solving some linear and non-linear BVPs of $10^{\text {th }}$ order is discussed in [8]. In $[9,10]$, the BVPs of $9^{\text {th }}$ and $10^{\text {th }}$ order are considered by adopting homotopy perturbation technique and the modified-variational iteration technique. Also the variational iterative technique was adopted in [11] for solving the $10^{\text {th }}$ order BVPs. Wazwaz [12-15] proposed modified form of ADM for solving $6^{\text {th }}, 8^{\text {th }}, 10^{\text {th }}$ and $12^{\text {th }}$ order.

The study of non-polynomial spline [16] of $11^{\text {th }}$ degree is a key element to solve $10^{\text {th }}$ order BVPs. In [17], it is depicted that the DEs that describe the $10^{\text {th }}$ order model to incorporate a 3rd order model of enlistment machine, two equations for dynamic power control, two equations for receptive power control, and three equations for edge pitch control. A $10^{\text {th }}$ order nonlinear dynamic model was developed in [18] to turn mobile robots that incorporate slip between the driven wheels and the ground. Based on binary six-point and eight-point approximating subdivision scheme, two collocation algorithms are constructed by $[19,20]$ to find the solution of BVPs. The $4^{\text {th }}$ order linear BVPs using a new cubic B-spline were solved in [21]. Authors explained the $10^{\text {th }}$ and $12^{\text {th }}$ order BVPs by using the Galerkin weighted residual technique in [22]. The $5^{\text {th }}, 6^{\text {th }}$ and $8^{\text {th }}$ order linear and non-linear BVPs by using the cubic B-spline scheme (CBS) method were solved in [23-25]. The higher $\left(10^{\text {th }}\right.$ and $\left.11^{\text {th }}\right)$ degree splines were tested in $[26,27]$ for solving $10^{\text {th }}$ order BVPs. In [28] they practiced 2nd order finite difference schemes for the mathematical solutions of the $8^{\text {th }}, 10^{\text {th }}$ and $12^{\text {th }}$ order Eigen-value problems. Galerkin method with septic B-spline and quintic B-spline was adopted in $[29,30]$ for solving $10^{\text {th }}$ order BVPs. Quintic B-spline and septic-B spline collocation methods was discussed in [31,32] to find solution of a $10^{\text {th }}$ order BVPs.

For discrete methods, e.g., Adomian decomposition, shooting, homotopy perturbation, finite differences and variational-iterative technique, only give discrete approximate values of the unknown $y(x)$. For fitting curve to data we require further data processing methods. To overcome these disadvantages, we introduced a new CBS scheme for the solution of $10^{\text {th }}$ order BVPs. The algorithm developed here is not only for the approximation solutions of the $10^{\text {th }}$ order boundary value problems(BVPs) employing CBS but also estimate derivatives of 1 st order to $10^{\text {th }}$ order (where boundary conditions (BCs) are defined) of the exact solution as well.

The rest of the paper is organized as follows. The construction of CBS is presented in Section 2. In Section 3, the CBS scheme is utilized as an interpolating function in the solution of $10^{\text {th }}$ order nonlinear BVPs. The results and discussion are presented in Section 4. Also some problems are considered in this section to show the efficiency of the CBS scheme. Finally, the concluding remarks are given in the final section.

\section{The Construction of CBS}

In this section, we construct the CBS basis functions for solving numerically the non-linear equations arising in the study of hydrodynamics and hydro-magnetic stability problems. To find the approximate solution at nodal points defined in the region $[a, b]$. For an interval $\Omega=[a, b]$, we divide it into $n$ sub-intervals $\Omega_{i}=\left[\kappa_{i}, \kappa_{i+1}\right] ; i=0,1,2, \ldots, n-1$, by the equidistant knots. For this range, we select equidistant points such that

$$
\Omega_{\imath}=\kappa_{\imath}=a+\imath h
$$

such that

$$
\Omega=\left\{a=\kappa_{0}, \ldots, \kappa_{n}=b\right\}
$$


i.e., $\kappa_{\imath}=a+\imath h,(\imath=0, \ldots, n)$ and $h=\frac{b-a}{n}$.

Assume $S_{3}(\Omega)=\left\{p(t) \in C^{2}[a, b]\right\}$ such that $p(t)$ converted to to cubic-polynomial on separately sub interval $\left(\kappa_{\imath}, \kappa_{l+1}\right)$. The basis function is defined as

$$
M_{l}(\kappa)=\frac{1}{6 h^{3}}\left\{\begin{array}{lc}
\left(\kappa-\kappa_{l-2}\right)^{3}, & \text { if } \kappa \in\left[\kappa_{l-2}, \kappa_{l-1}\right], \\
h^{3}+3 h^{2}\left(\kappa-\kappa_{l-1}\right)+3 h\left(\kappa-\kappa_{l-1}\right)^{2}-3 & \text { if } \kappa \in\left[\kappa_{l-1}, \kappa_{l}\right], \\
\left(\kappa-\kappa_{l-1}\right)^{3}, & \text { if } \kappa \in\left[\kappa_{l}, \kappa_{l+1}\right], \\
h^{3}+3 h^{2}\left(\kappa_{l+1}-\kappa\right)+3 h\left(\kappa_{l+1}-\kappa\right)^{2}-3 & \text { if } \kappa \in\left[\kappa_{l+1}, \kappa_{l+2}\right], \\
\left(\kappa_{l+2}-\kappa\right)^{3}, & \text { otherwise, }
\end{array}\right.
$$

for $(\imath=2,3,4, \ldots, n-2)$. Considering one and all $M_{l}(\kappa)$ is also a piece-wise cubic with knots at $\Omega$, simultaneously $M_{l}(\kappa) \in S_{3}(\Omega)$.

Assume $\Psi=\left\{M_{\imath}\right\} ; \quad(\imath=-1,0,1,2 \ldots n, n+1)$ be linearly independent and let $M_{3}(\Omega)=\operatorname{span} \Psi$. Thus $M_{3}(\Omega)$ is $(n+3)$ dimensional and $M_{3}(\Omega)=S_{3}(\Omega)$. Let $s(\kappa)$ be the cubic-B spline function interpolating at the nodal points and $s(\kappa) \in S_{3}(\Omega)$. Then $s(\kappa)$ can be written as

$$
s(\kappa)=\sum_{i=-1}^{n+1} j_{l} M_{l}(\kappa)
$$

Consequently now for a function $w(\kappa)$, there happened to be a distinctive cubic-B spline $s(\kappa)=\sum_{l=-1}^{n+1} \jmath_{l} M_{l}(\kappa)$, satisfying the interpolating conditions:

$$
w\left(\kappa_{\imath}\right)=s\left(\kappa_{\imath}\right)=\frac{\jmath_{l-1}+4 \jmath_{l}+\jmath_{l+1}}{6},
$$

for $\imath=0, \ldots, n$.

The values of $M_{l}(\kappa)$, and its derivatives $M_{l}^{(1)}(\kappa), M_{l}^{(2)}(\kappa)$ at nodal points are required and these derivatives are tabulated in Table 1.

Table 1. Values of $M_{l}(\kappa)$ and its derivatives.

\begin{tabular}{llll}
\hline & $\boldsymbol{M}_{\boldsymbol{\imath}}(\boldsymbol{\kappa})$ & $\boldsymbol{M}_{\boldsymbol{i}}{ }^{(1)}(\boldsymbol{\kappa})$ & $\boldsymbol{M}_{\boldsymbol{\imath}}{ }^{(2)}(\kappa)$ \\
\hline$\kappa_{l-2}, \kappa_{\imath+2}$ & 0 & 0 & 0 \\
$\kappa_{l-1}$ & $1 / 6$ & $1 / 2 h$ & $1 / h^{2}$ \\
$\kappa_{l}$ & $4 / 6$ & 0 & $-2 / h^{2}$ \\
$\kappa_{l+1}$ & $1 / 6$ & $-1 / 2 h$ & $1 / h^{2}$ \\
otherwise & 0 & 0 & 0 \\
\hline
\end{tabular}

Assume $m_{l}=s^{(1)}\left(\kappa_{l}\right)$ and $\aleph_{l}=s^{(2)}\left(\kappa_{l}\right)$ then from

$$
\begin{gathered}
m_{\imath}=s^{(1)}\left(\kappa_{\imath}\right)=w^{(1)}\left(\kappa_{\imath}\right)-\frac{1}{180} h^{4} w^{(5)}\left(\kappa_{\imath}\right)+O\left(h^{6}\right) \\
w^{(1)}(\kappa)=s^{(1)}\left(\kappa_{\imath}\right)=\frac{\jmath_{l+1}-\jmath_{l-1}}{2 h} \\
\aleph_{\iota}=s^{(2)}\left(\kappa_{l}\right)=w^{(2)}\left(\kappa_{\imath}\right)-\frac{1}{12} h^{2} w^{(4)}\left(\kappa_{\imath}\right)+\frac{1}{360} h^{4} w^{(6)}\left(\kappa_{l}\right)+O\left(h^{6}\right)
\end{gathered}
$$




$$
w^{(2)}(\kappa)=s^{(2)}\left(\kappa_{l}\right)=\frac{\jmath_{l+1}-2 \jmath_{l}+\jmath_{l-1}}{h^{2}},
$$

$\aleph_{\imath}$ may be used to determine numerical-difference formulas for $w^{(3)}\left(\kappa_{\imath}\right), w^{(4)}\left(\kappa_{\imath}\right)$ such that $(l=1$ to $n-$ $1)$, for $w^{(5)}\left(\kappa_{l}\right), w^{(6)}\left(\kappa_{l}\right)$ such that $(l=2$ to $n-2)$, for $w^{(7)}\left(\kappa_{l}\right), w^{(8)}\left(\kappa_{l}\right)$ such that $(l=3$ to $n-3)$ and $w^{(9)}\left(\kappa_{l}\right), w^{(10)}\left(\kappa_{l}\right)$ such that $(\imath=4$ to $n-4)$ like so the errors can be obtained by using Taylor-series

$$
\left\{\begin{array}{c}
\frac{\aleph_{l+1}-\aleph_{l-1}}{2 h}=\frac{s^{(3)}\left(\kappa_{l-}\right)+s^{(3)}\left(\kappa_{l+}\right)}{2}=w^{(3)}\left(\kappa_{\imath}\right)+\frac{1}{12} h^{2} w^{(5)}\left(\kappa_{\imath}\right)+O\left(h^{4}\right) \\
w^{(3)}(\kappa)=s^{(3)}\left(\kappa_{l}\right)=\frac{\jmath_{l+2}-2 \jmath_{l}+1+2 \jmath_{l-1}-\jmath_{l-2}}{2 h^{3}} \\
\frac{\aleph_{l+1}-2 \aleph_{l}+\aleph_{l-1}}{h^{2}}=\frac{s^{(3)}\left(\kappa_{l-}\right)-s^{(3)}\left(\kappa_{l+}\right)}{h}=w^{(4)}\left(\kappa_{\imath}\right)-\frac{1}{720} h^{4} w^{(8)}\left(\kappa_{l}\right)+O\left(h^{6}\right) \\
w^{(4)}(\kappa)=s^{(4)}\left(\kappa_{\imath}\right)=\frac{\jmath_{l+2}-4 \jmath_{l+1}+6 \jmath_{l}-4 \jmath_{l-1}+\jmath_{l-2}}{h^{4}} \\
\frac{\aleph_{l+2}-2 \aleph_{l+1}+2 \aleph_{l-1}-\aleph_{l-2}=w^{(5)}\left(\kappa_{\imath}\right)+O\left(h^{2}\right)}{2 h^{3}} \\
w^{(5)}(\kappa)=s^{(5)}\left(\kappa_{l}\right)=\frac{\jmath_{l+3}-4 \jmath_{l+2}+5 j_{l+1}+5 \jmath_{l-1}+4 \jmath_{l-2}-\jmath_{l-3}}{2 h^{5}}
\end{array}\right.
$$

Similarly (see [31]),

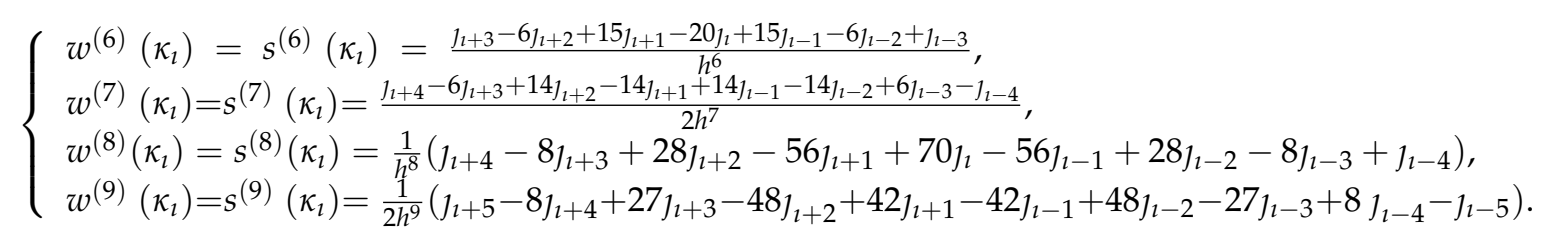

\section{The $10^{\text {th }}$ Order Nonlinear BVPs}

In this section, we consider the $10^{\text {th }}$ order nonlinear BVPs arising in the study of hydrodynamics stability and visco-elastic flows.

$$
\begin{aligned}
& w^{(10)}(\kappa)=f\left(\kappa, w(\kappa), w^{(1)}(\kappa), w^{(2)}(\kappa), w^{(3)}(\kappa), w^{(4)}(\kappa), w^{(5)}(\kappa), w^{(6)}(\kappa), w^{(7)}(\kappa)\right. \\
& \left.w^{(8)}(\kappa), w^{(9)}(\kappa)\right), \kappa \in[a, b]
\end{aligned}
$$

with BCs

$$
\begin{array}{lcc}
w(a)=\lambda_{0}, & w^{(1)}(a)=\lambda_{1}, & w^{(2)}(a)=\lambda_{2}, \\
w^{(3)}(a)=\lambda_{3}, & w^{(4)}(a)=\lambda_{4}, & w(b)=\chi_{0}, \\
w^{(1)}(b)=\chi_{1}, & w^{(2)}(b)=\chi_{2}, & w^{(3)}(b)=\chi_{3} \\
w^{(4)}(b)=\chi_{4}, &
\end{array}
$$

where $\lambda_{0}, \lambda_{1}, \lambda_{2}, \lambda_{3}, \lambda_{4}$ and $\chi_{0}, \chi_{1}, \chi_{2}, \chi_{3}, \chi_{4}$ are given real constants, $\left(a_{\imath}(\kappa) ; \imath=1,2, \ldots, 10\right)$ and $f$ is continuous in interval $[a, b]$.

The Taylor, series for $w^{(10)}\left(\kappa_{l}\right)$ at the preferred collocation points alongside central difference (see [31]), we have

$$
\begin{aligned}
& w^{(10)}\left(\kappa_{\imath}\right)=\frac{1}{h^{6}}\left(w_{l+3}{ }^{(4)}\left(\kappa_{\imath}\right)-6 w_{l+2}{ }^{(4)}\left(\kappa_{l}\right)+15 w_{l+1}{ }^{(4)}\left(\kappa_{l}\right)-20 w_{l}{ }^{(4)}\left(\kappa_{\imath}\right)+15\right. \\
& \left.w_{l-1}{ }^{(4)}\left(\kappa_{\imath}\right)-6 w_{l-2}{ }^{(4)}\left(\kappa_{l}\right)+w_{l-3}{ }^{(4)}\left(\kappa_{l}\right)\right) .
\end{aligned}
$$

Equation (9) can be written as 


$$
\begin{aligned}
& \frac{\aleph_{l-2}-2 \aleph_{l-3}+\aleph_{l-4}}{h^{2}}=w^{(4)}\left(\kappa_{l-3}\right)-\frac{1}{720} h^{4} w^{(8)}\left(\kappa_{l-3}\right)+O\left(h^{6}\right), \\
& \frac{\aleph_{l-1}-2 \aleph_{l-2}+\aleph_{l-3}}{h^{2}}=w^{(4)}\left(\kappa_{l-2}\right)-\frac{1}{720} h^{4} w^{(8)}\left(\kappa_{l-2}\right)+O\left(h^{6}\right), \\
& \frac{\aleph_{l}-2 \aleph_{l-1}+\aleph_{l-2}}{h^{2}}=w^{(4)}\left(\kappa_{l-1}\right)-\frac{1}{720} h^{4} w^{(8)}\left(\kappa_{l-1}\right)+O\left(h^{6}\right), \\
& \frac{\aleph_{l+2}-2 \aleph_{l+1}+\aleph_{l}}{h^{2}}=w^{(4)}\left(\kappa_{l+1}\right)-\frac{1}{720} h^{4} w^{(8)}\left(\kappa_{l+1}\right)+O\left(h^{6}\right), \\
& \frac{\aleph_{l+3}-2 \aleph_{l+2}+\aleph_{l+1}}{h^{2}}=w^{(4)}\left(\kappa_{l+2}\right)-\frac{1}{720} h^{4} w^{(8)}\left(\kappa_{l+2}\right)+O\left(h^{6}\right), \\
& \frac{\aleph_{l+4}-2 \aleph_{l+3}+\aleph_{l+2}}{h^{2}}=w^{(4)}\left(\kappa_{l+3}\right)-\frac{1}{720} h^{4} w^{(8)}\left(\kappa_{l+3}\right)+O\left(h^{6}\right) .
\end{aligned}
$$

Substituting Equation (13) into Equation (12), we obtain

$$
\begin{aligned}
& \frac{1}{h^{8}}\left(\aleph_{l+4}-8 \aleph_{l+3}+28 \aleph_{l+2}-56 \aleph_{l+1}+70 \aleph_{l}-56 \aleph_{l-1}+28 \aleph_{l-2}-8 \aleph_{l-3}+\aleph_{l-4}\right) \\
& =w^{(10)}\left(\kappa_{l}\right)+O\left(h^{2}\right) .
\end{aligned}
$$

Since $\aleph_{l}=\frac{\jmath_{l+1}-2 \jmath_{l}+\jmath_{l}-1}{h^{2}}$ so, Equation (14) becomes

$$
\begin{aligned}
& w^{(10)}\left(\kappa_{l}\right)=\frac{1}{h^{8}}\left(\frac{\jmath_{l+5}-2 \jmath_{l+4}+\jmath_{l+3}}{h^{2}}-8\left(\frac{\jmath_{l+4}-2 \jmath_{l+3}+\jmath_{l+2}}{h^{2}}\right)+28\left(\frac{\jmath_{l+3}-2 \jmath_{l+2}+\jmath_{l+1}}{h^{2}}\right)-56\left(\frac{\jmath_{l+2}-2 \jmath_{l+1}+\jmath_{l}}{h^{2}}\right)\right. \\
& +70\left(\frac{\jmath_{l+1}-2 \jmath_{l}+\jmath_{l-1}}{h^{2}}\right)-56\left(\frac{\jmath_{l}-2 \jmath_{l-1}+\jmath_{l-2}}{h^{2}}\right)+28\left(\frac{\jmath_{l-1}-2 \jmath_{l-2}+\jmath_{l-3}}{h^{2}}\right)-8\left(\frac{\jmath_{l-2}-2 \jmath_{l-3}+\jmath_{l-4}}{h^{2}}\right)+ \\
& \left.\frac{\jmath_{l-3}-2 \jmath_{l-4}+\jmath_{l-5}}{h^{2}}\right) .
\end{aligned}
$$

After some simplifications the above equation becomes

$$
\begin{aligned}
& w^{(10)}\left(\kappa_{\iota}\right)=S^{(10)}\left(\kappa_{\imath}\right)=\frac{1}{h^{10}}\left(\jmath_{l+5}-10 \jmath_{l+4}+45 \jmath_{l+3}-120 \jmath_{l+2}+210 \jmath_{l+1}-252 \jmath_{l}\right. \\
& \left.+210 \jmath_{l-1}-120 \jmath_{l-2}+45 \jmath_{l-3}-10 \jmath_{l-4}+\jmath_{l-5}\right) .
\end{aligned}
$$

Let $w\left(\kappa_{l}\right)=s\left(\kappa_{l}\right)=\sum_{l=-1}^{n+1} \jmath_{l} M_{l}\left(\kappa_{l}\right)$ be the accurate solution of non-linear $10^{\text {th }}$ order BVPs

$$
\begin{aligned}
& w^{(10)}\left(\kappa_{l}\right)=f\left(\kappa_{l}, w\left(\kappa_{l}\right), w^{(1)}\left(\kappa_{l}\right), w^{(2)}\left(\kappa_{\imath}\right), w^{(3)}\left(\kappa_{l}\right), w^{(4)}\left(\kappa_{l}\right),\right. \\
& \left.w^{(5)}\left(\kappa_{l}\right), w^{(6)}\left(\kappa_{l}\right), w^{(7)}\left(\kappa_{l}\right), w^{(8)}\left(\kappa_{l}\right), w^{(9)}\left(\kappa_{l}\right)\right), \kappa_{l} \in[a, b] .
\end{aligned}
$$

Imposing Equations (3), (5), (7), (8) and (9) into Equation (17), we have

$$
\begin{aligned}
& \frac{1}{h^{10}}\left(\jmath_{l+5}-10 \jmath_{l+4}+45 \jmath_{l+3}-120 \jmath_{l+2}+210 \jmath_{l+1}-252 \jmath_{l}+210 \jmath_{l-1}-120 \jmath_{l-2}+45 \jmath_{l-3}\right. \\
& \left.-10 \jmath_{l-4}+\jmath_{l-5}\right)=f_{l}\left(\kappa_{l}, \frac{1}{6}\left(\jmath_{l-1}+4 \jmath_{l}+\jmath_{l+1}\right), \frac{1}{2 h}\left(\jmath_{l+1}-\jmath_{l-1}\right), \frac{1}{h^{2}}\left(\jmath_{l+1}-2 \jmath_{l}+\jmath_{l-1}\right),\right. \\
& \frac{1}{2 h^{3}}\left(\jmath_{l+2}-2 \jmath_{l+1}+2 \jmath_{l-1}-\jmath_{l-2}\right), \frac{1}{h^{4}}\left(\jmath_{l+2}-4 \jmath_{l+1}+6 \jmath_{l}-4 \jmath_{l-1}+\jmath_{l-2}\right), \frac{1}{2 h^{5}}\left(\jmath_{l+3}\right. \\
& \left.-4 \jmath_{l+2}+5 \jmath_{l+1}+5 \jmath_{l-1}+4 \jmath_{l-2}-\jmath_{l-3}\right), \frac{1}{h^{6}}\left(\jmath_{l+3}-6 \jmath_{l+2}+15 \jmath_{l+1}-20 \jmath_{l}+15 \jmath_{l-1}\right. \\
& \left.-6 \jmath_{l-2}+\jmath_{l-3}\right), \frac{1}{2 h^{7}}\left(\jmath_{l+4}-6 \jmath_{l+3}+14 \jmath_{l+2}-14 \jmath_{l+1}+14 \jmath_{l-1}-14 \jmath_{l-2}+6 \jmath_{l-3}-\jmath_{l-4}\right), \\
& \frac{1}{h^{8}}\left(\jmath_{l+4}-8 \jmath_{l+3}+28 \jmath_{l+2}-56 \jmath_{l+1}+70 \jmath_{l}-56 \jmath_{l-1}+28 \jmath_{l-2}-8 \jmath_{l-3}+\jmath_{l-4}\right), \frac{1}{2 h^{9}}\left(\jmath_{l+5}-8 \jmath_{l+4}\right. \\
& \left.\left.+27 \jmath_{l+3}-48 \jmath_{l+2}+42 \jmath_{l+1}-42 \jmath_{l-1}+48 \jmath_{l-2}-27 \jmath_{l-3}+8 \jmath_{l-4}-\jmath_{l-5}\right)\right), \kappa \in[a, b] .
\end{aligned}
$$


Equation (18) we will produce a new system consisting of $(n-7)$ linear equations $(\imath=4,5, \ldots, n-4)$ with $(n+3)$ unknowns $\jmath_{l}$ where $(\imath=-1,0, \ldots, n+1)$, therefore ten further equations are required. From given $\mathrm{BCs}$ at $\kappa=a$, we have five equations:

$$
\begin{aligned}
& w(a)=\lambda_{0} \Rightarrow \quad{ }_{-1}+4 \jmath_{0}+\jmath_{1}=6 \lambda_{0} \\
& w^{(1)}(a)=\lambda_{1} \Rightarrow \quad-\jmath_{-1}+\jmath_{1}=2 \lambda_{1} h \\
& w^{(2)}(a)=\lambda_{2} \Rightarrow \jmath_{-1}-2 \jmath_{0}+\jmath_{1}=\lambda_{2} h^{2} \\
& w^{(3)}(a)=\lambda_{3} \Rightarrow \jmath_{2}-2 \jmath_{1}+2 \jmath_{-1}-\jmath_{-2}=2 \lambda_{3} h^{3} \\
& w^{(4)}(a)=\lambda_{4} \Rightarrow \jmath_{2}-4 \jmath_{1}+6 \jmath_{0}-4 \jmath_{-1}+\jmath_{-2}=\lambda_{4} h^{4},
\end{aligned}
$$

similarly from $\kappa=b$ there will be other five equations

$$
\begin{aligned}
& w(b)=\chi_{0} \Rightarrow \quad \jmath_{n-1}+4 \jmath_{n}+\jmath_{n+1}=6 \chi_{0} \\
& w^{(1)}(b)=\chi_{1} \Rightarrow \quad-\jmath_{n-1}+\jmath_{n+1}=2 \chi_{1} h \\
& w^{(2)}(b)=\chi_{2} \Rightarrow \quad \jmath_{n-1}-2 \jmath_{n}+\jmath_{n+1}=\chi_{2} h^{2} \\
& w^{(3)}(b)=\chi_{3} \Rightarrow \quad \jmath_{n+2}-2 \jmath_{n+1}+2 \jmath_{n-1}-\jmath_{n-2}=2 \chi_{3} h^{3} \\
& w^{(4)}(b)=\chi_{4} \Rightarrow \quad \jmath_{n+2}-4 \jmath_{n+1}+6 \jmath_{n}-4 \jmath_{n-1}+\jmath_{n-2}=\chi_{4} h^{4} .
\end{aligned}
$$

Omitting the order of the error of terms, the exact solution $w\left(\kappa_{l}\right)=s\left(\kappa_{l}\right)=\sum_{l=-1}^{n+1} \jmath_{l} M_{l}\left(\kappa_{l}\right)$ is accomplished by finding solution of the discussed above linear system of $(n+3)$ equations in $(n+3)$ unknowns considering the Equations (18)-(20).

\section{Convergence Analysis}

Let $\widehat{w}(\kappa)$ be the exact solution of the Equations (10)-(12) and also $\widehat{s}(\kappa)$ be the CBS approximation to $\widehat{w}(\kappa)$. Therefore, we have

$$
\widehat{w}\left(\kappa_{\imath}\right)=\widehat{s}\left(\kappa_{\imath}\right)=\sum_{l=-1}^{n+1} \widehat{\jmath}_{\imath} M_{\imath}\left(\kappa_{\imath}\right)
$$

where

$$
\widehat{\jmath}=\widehat{\jmath}_{\text {imath }}=\left[\widehat{\jmath}_{-1}, \widehat{\jmath}_{0}, \widehat{\jmath}_{1}, \ldots, \widehat{\jmath}_{n+1}\right]^{T} .
$$

Also, we have assume that $s^{\prime}(\kappa)$ be the computed cubic B spline approximation to $\widehat{s}(\kappa)$, namely

$$
\begin{gathered}
w^{\prime}\left(\kappa_{\imath}\right)=s^{\prime}\left(\kappa_{\imath}\right)=\sum_{i=-1}^{n+1} \jmath_{i}^{\prime} M_{l}\left(\kappa_{\imath}\right), \\
\jmath^{\prime}=\jmath_{i}^{\prime}=\left[\jmath^{\prime}{ }_{-1}, \jmath^{\prime}{ }_{0}, \jmath^{\prime}{ }_{1}, \ldots, \jmath_{n+1}^{\prime}\right]^{T} .
\end{gathered}
$$

To approximate the error $\left.\left.\| \widehat{w}\left(\kappa_{l}\right)\right)-\widehat{s}\left(\kappa_{l}\right)\right) \|_{\infty}$ we have to estimate error $\left.\left.\| \widehat{w}\left(\kappa_{l}\right)\right)-s^{\prime}\left(\kappa_{l}\right)\right) \|_{\infty}$ and $\left.\left.\| w^{\prime}\left(\kappa_{l}\right)\right)-\widehat{s}\left(\kappa_{\imath}\right)\right) \|_{\infty}$ seperately

The system of $(n+3) \times(n+3)$ matrix can be written as:

$$
B_{j}=G \text {. }
$$

Then, we have

$$
B \widehat{\jmath}=\widehat{G}
$$

and

$$
B \jmath^{\prime}=G^{\prime}
$$


Now, by subtracting Equations (22) and (23), we obtain

$$
B\left(\jmath^{\prime}-\widehat{\jmath}\right)=G^{\prime}-\widehat{G},
$$

where $B$ is an $(n+3) \times(n+3)$-dimensional band matrix, and

$$
G=\left[G_{-1}, G_{0}, G_{1}, \ldots, G_{n+1}\right]^{T}
$$

where $T$ denoting transpose.

We can write

$$
\left(\jmath^{\prime}-\widehat{\jmath}\right)=B^{-1}\left(G^{\prime}-\widehat{G}\right) .
$$

Taking the infinity norm from Equation (24), we obtain

$$
\left\|\left(\jmath^{\prime}-\widehat{\jmath}\right)\right\|_{\infty}=\left\|B^{-1}\right\|_{\infty}\left\|G^{\prime}-\widehat{G}\right\|_{\infty} .
$$

The B-spline $M=M_{l}=\left\{M_{-1}, M_{0}, M_{1}, \ldots, M_{n+1}\right\}$ satisfy the following property

$$
\left|\sum_{i=-1}^{n+1} J_{i}^{\prime} M_{\imath}\left(\kappa_{l}\right)\right| \leq 1
$$

Using [24]

$$
\begin{gathered}
\left\|B^{-1}\right\|_{\infty}\left\|G^{\prime}-\widehat{G}\right\|_{\infty} \leq b h^{2} . \\
\left\|\left(\jmath^{\prime}-\widehat{\jmath}\right)\right\|_{\infty} \leq b h^{2} . \\
\left.s^{\prime}\left(\kappa_{\imath}\right)\right)-\widehat{s}\left(\kappa_{l}\right)=\left(\jmath^{\prime}-\widehat{\jmath}\right) \sum_{i=-1}^{n+1} M_{l}\left(\kappa_{l}\right) . \\
\left\|s^{\prime}\left(\kappa_{l}\right)-\widehat{s}\left(\kappa_{l}\right)\right\|_{\infty}=\left\|\left(\jmath^{\prime}-\widehat{\jmath}\right) \sum_{i=-1}^{n+1} M_{l}\left(\kappa_{l}\right)\right\|_{\infty} . \\
\left\|s^{\prime}\left(\kappa_{l}\right)-\widehat{s}\left(\kappa_{\imath}\right)\right\|_{\infty} \leq\left\|\left(\jmath^{\prime}-\widehat{\jmath}\right)\right\|_{\infty}\left|\sum_{i=-1}^{n+1} M_{l}\left(\kappa_{l}\right)\right| \leq b h^{2} . \\
\left\|\widehat{w}\left(\kappa_{l}\right)-s^{\prime}\left(\kappa_{l}\right)\right\|_{\infty} \leq \rho h^{4} . \\
\left\|\widehat{w}\left(\kappa_{l}\right)-\widehat{s}\left(\kappa_{l}\right)\right\|_{\infty} \leq\left\|\widehat{w}\left(\kappa_{l}\right)-s^{\prime}\left(\kappa_{l}\right)\right\|_{\infty}+\left\|s^{\prime}\left(\kappa_{l}\right)-\widehat{s}\left(\kappa_{l}\right)\right\|_{\infty} .
\end{gathered}
$$

Using Equations (26) and (27) in Equation (28)

$$
\left\|\widehat{w}\left(\kappa_{l}\right)-\widehat{s}\left(\kappa_{l}\right)\right\|_{\infty} \leq b h^{2}+\rho h^{4}=\ell h^{2} .
$$

which proves that this method is second order convergent and $\|\widehat{w}(\kappa)-\widehat{s}(\kappa)\|_{\infty} \leq \ell h^{2}$.

\section{Results and Discussions}

To test the accuracy of CBS method, three problems are discussed and compared with the existing methods in this section.

\subsection{Problem 1}

We consider the following DEs arising in viscoelastic flows and hydrodynamic stability problems as given in $[29,31]$

$$
w^{(10)}(\kappa)=\frac{14175}{4}(\jmath+w(\kappa)+1)^{11} ; 0 \leq \kappa \leq 1 ;
$$


subject to BCs;

$$
\begin{gathered}
w(0)=w(1)=0, \quad w^{(1)}(0)=-\frac{1}{2}=-w^{(2)}(0), \quad w^{(1)}(1)=1, \\
w^{(2)}(1)=4, \quad w^{(3)}(0)=\frac{3}{4}, \quad w^{(3)}(1)=12, \quad w^{(4)}(0)=\frac{3}{2}, \quad w^{(4)}(1)=48 .
\end{gathered}
$$

the exact solution of given equation is $w(\kappa)=\frac{2}{2-\kappa}-\kappa-1$. The values of fifteen unknowns $j_{i}$ from the Equations (18)-(20) are

$$
\begin{array}{lll}
J_{-2}=0.10849167, & J_{3}=-0.13626667, \\
J_{-1}=0.05166667, & J_{4}=-0.15061956, & J_{9}=-0.08666667, \\
J_{0}=-0.00083333, & J_{5}=-0.16684713, & J_{11}=0.0 .0066667, \\
J_{1}=-0.048333333, & J_{6}=-0.17169449, & J_{12}=0.28773333, \\
J_{2}=-0.09000833, & J_{7}=-0.16277005, &
\end{array}
$$

Tables 2 and 3 analyzed the exact solution and cubic B-spline scheme (CBS) solution of problem 1 at $h=\frac{1}{10}$ and $h=\frac{1}{5}$ respectively. Figures 1-3 analyze the exact solution with cubic B-spline scheme (CBS) solution of problem 1 at $h=\frac{1}{10}$ and $h=\frac{1}{5}$ graphically. Table 4 analyze the errors at those derivatives where boundary conditions (BCs) are defined in problem 1 at $h=\frac{1}{10}$.

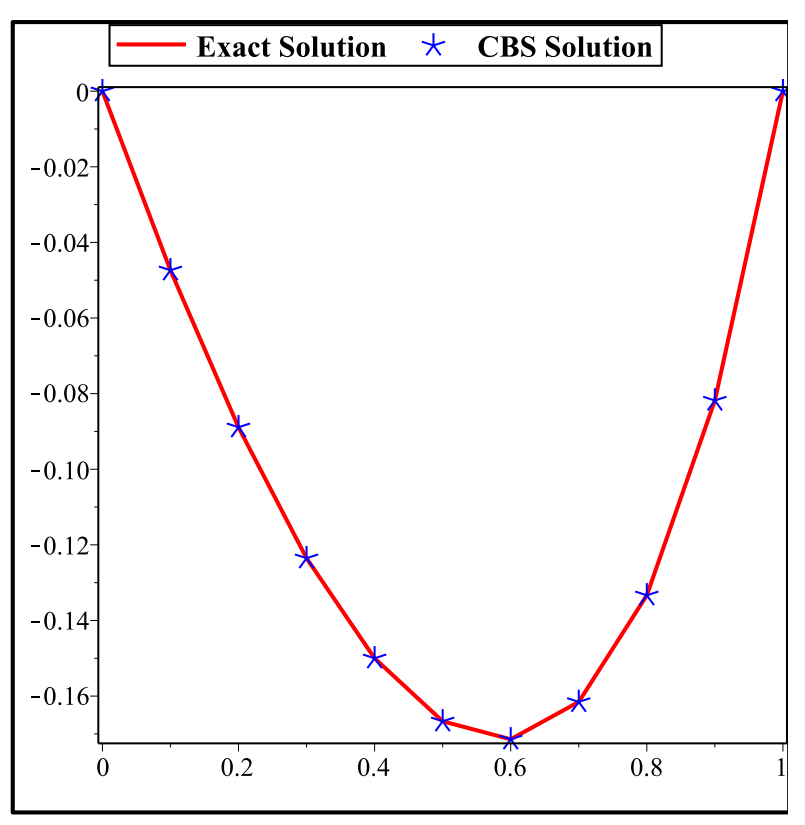

Figure 1. Problem 1 at $h=\frac{1}{10}$. 
Table 2. Analyzing exact solution and cubic B-spline scheme (CBS) solution of problem 1 at $h=\frac{1}{10}$.

\begin{tabular}{llll}
\hline $\boldsymbol{\kappa}$ & Exact Solution & CBS Solution & Absolute Error \\
\hline 0 & 0 & 0 & $0 \times 10^{0}$ \\
0.1 & -0.0473684 & -0.0473665 & $1.900 \times 10^{-06}$ \\
0.2 & -0.0888889 & -0.0888822 & $6.670 \times 10^{-05}$ \\
0.3 & -0.1235294 & -0.1235488 & $3.810 \times 10^{-05}$ \\
0.4 & -0.1500000 & -0.1509819 & $1.020 \times 10^{-04}$ \\
0.5 & -0.1666667 & -0.1669504 & $1.720 \times 10^{-04}$ \\
0.6 & -0.1714286 & -0.1714992 & $2.030 \times 10^{-05}$ \\
0.7 & -0.1615385 & -0.1615302 & $1.700 \times 10^{-06}$ \\
0.8 & -0.1333333 & -0.1333172 & $9.160 \times 10^{-05}$ \\
0.9 & -0.0818182 & -0.0818000 & $2.180 \times 10^{-05}$ \\
1 & 0 & 0 & $0 \times 10^{0}$ \\
\hline
\end{tabular}

Table 3. Analyzing exact solution and CBS solution of problem 1 at $h=\frac{1}{5}$.

\begin{tabular}{llll}
\hline $\boldsymbol{\kappa}$ & Exact Solution & CBS Solution & Absolute Error of CBS \\
\hline 0 & 0 & 0 & $0 \times 10^{0}$ \\
0.2 & -0.0888889 & -0.0888000 & $8.890 \times 10^{-05}$ \\
0.4 & -0.1500000 & -0.1500222 & $2.980 \times 10^{-05}$ \\
0.6 & -0.1714286 & -0.1714778 & $1.150 \times 10^{-05}$ \\
0.8 & -0.1333333 & -0.1333000 & $3.730 \times 10^{-05}$ \\
1 & 0 & 0 & $0 \times 10^{0}$ \\
\hline
\end{tabular}

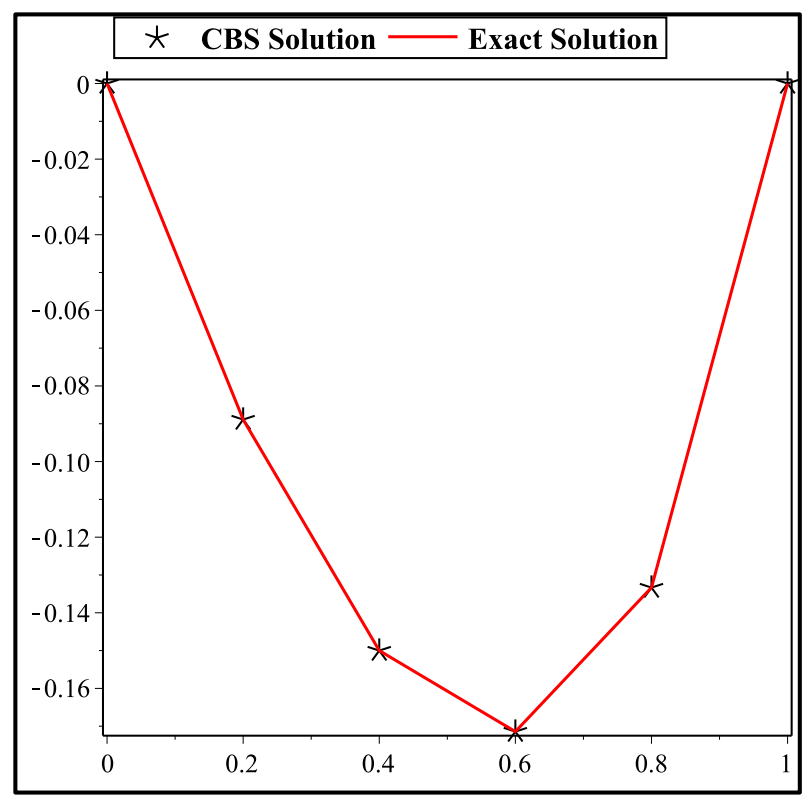

Figure 2. Problem 1 at $h=\frac{1}{5}$. 


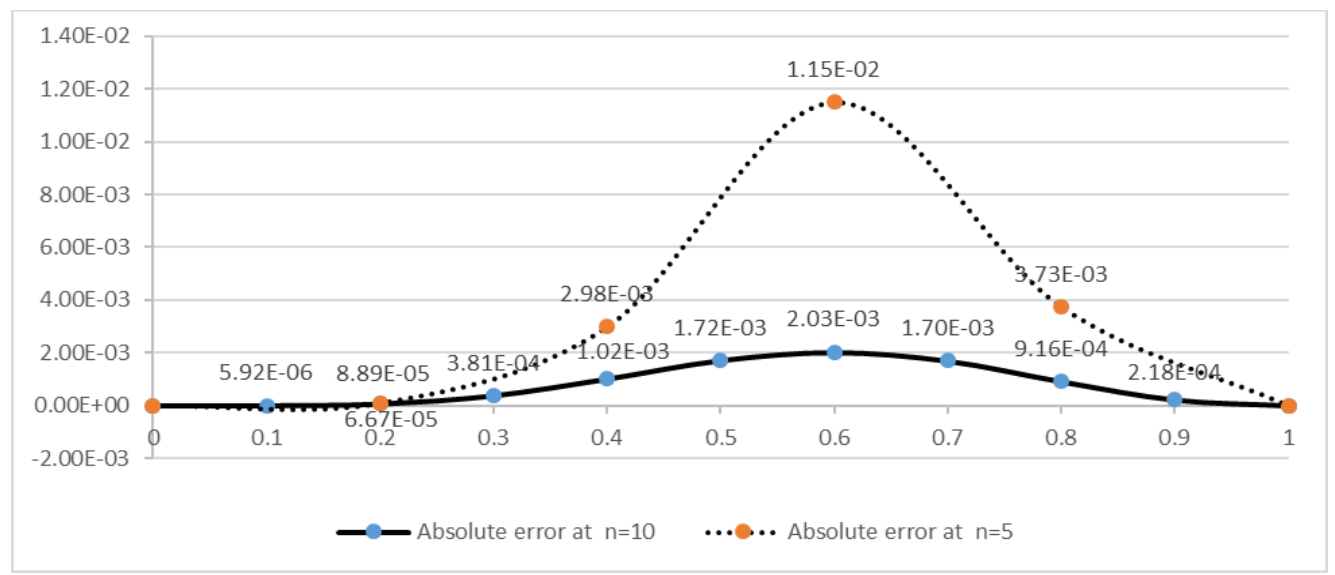

Figure 3. Problem 1 at $h=\frac{1}{10}$ and $h=\frac{1}{5}$.

Table 4. Errors at derivatives where boundary conditions (BCs) are defined in problem 1 at $h=\frac{1}{10}$.

\begin{tabular}{lllll}
\hline $\boldsymbol{\kappa}$ & $\begin{array}{l}\text { CBS-Solution } \\
\boldsymbol{w}^{(\mathbf{1})}(\boldsymbol{\kappa})\end{array}$ & $\begin{array}{l}\text { CBS-Solution } \\
\boldsymbol{w}^{(\mathbf{2})}(\boldsymbol{\kappa})\end{array}$ & $\begin{array}{l}\text { of } \\
\boldsymbol{w}^{(3)}(\boldsymbol{\kappa})\end{array}$ & $\begin{array}{l}\text { CBS-Solution of } \\
\boldsymbol{w}^{(\mathbf{4})}(\boldsymbol{\kappa})\end{array}$ \\
\hline 0 & -0.5 & 0.5 & 0.75 & 1.5 \\
0.1 & -0.4459 & 0.5825 & 1.0585 & 1.93853 \\
0.2 & -0.3812 & 0.7117 & 1.3398 & 2.54026 \\
0.3 & -0.3031 & 0.8505 & 1.3543 & 3.38062 \\
0.4 & -0.2114 & 0.9826 & 1.4378 & 4.57764 \\
0.5 & -0.1054 & 1.1380 & 1.9730 & 6.32099 \\
0.6 & 0.0204 & 1.3772 & 3.0994 & 8.92485 \\
0.7 & 0.1771 & 1.7579 & 4.6624 & 12.92780 \\
0.8 & 0.3805 & 2.3097 & 6.4105 & 19.29012 \\
0.9 & 0.6480 & 3.0400 & 8.4517 & 29.80422 \\
1 & 1 & 4 & 12 & 48 \\
\hline
\end{tabular}

\subsection{Problem 2}

We consider the following problem as given in [16]

$$
w^{(10)}(\kappa)=9 !\left(e^{-10 w(\kappa)}-\frac{2}{(1+\kappa)^{10}}\right) ; 0 \leq \kappa \leq e^{1 / 2-1}
$$

subject to BCs;

$$
\begin{gathered}
w(0)=0, \quad \begin{array}{c}
w\left(e^{1 / 2-1}\right)=\frac{1}{2}, \quad w^{(1)}(0)=-w^{(2)}(0)=1, \quad w^{(1)}\left(e^{1 / 2-1}\right)=e^{\left(\frac{-1}{2}\right),} \\
w^{(2)}\left(e^{1 / 2-1}\right)=-e^{(-1)}, \quad w^{(3)}(0)=2, \quad w^{(3)}\left(e^{1 / 2-1}\right)=2 e^{\left(-\frac{3}{2}\right)}, \\
w^{(4)}(0)=-6, \quad w^{(4)}\left(e^{1 / 2-1}\right)=-6 e^{(-2)},
\end{array}
\end{gathered}
$$

the exact solution of a given equation is $w(\kappa)=\ln (1+\kappa)$ where the domain $\left[0, e^{1 / 2-1}\right]$ for $h=$ $2^{-i} e^{1 / 2-1}$

The values of fifteen unknowns $j_{i}$ from Equations (18)-(20) are 


$$
\begin{aligned}
& \jmath-2=-0.13805879, \\
& \jmath_{-1}=-0.0662749, \\
& \jmath_{0}=0.0007014, \\
& \jmath_{1}=0.0634693, \\
& \jmath_{2}=0.1225218,
\end{aligned}
$$$$
\jmath_{3}=0.1782805,
$$$$
J_{4}=0.2311044 \text {, }
$$

${ }_{8}=0.4183388$,

$19=0.4601370$,

$\jmath_{10}=0.5002580$,

$J_{6}=0.3290924$,

$J_{11}=0.5388309$,

$17=0.3747130$,

Tables 5 and 6 analyzed the exact solution and cubic B-spline scheme (CBS) solution of problem 2 at $h=0.064872$ and $h=0.12974426$ respectively. Figures $4-6$ analyze the exact solution with cubic B-spline scheme (CBS) solution of problem 2 at $h=0.064872$ and $h=0.12974426$ graphically. Table 7 analyze the errors at those derivatives where boundary conditions (BCs) are defined in problem 2 at $h=0.064872$.

Table 5. Analyzing exact solution and CBS-solution of problem 2 at $h=0.064872$.

\begin{tabular}{llll}
\hline $\boldsymbol{\kappa}$ & Exact Solution & CBS Solution & Absolute Error of CBS \\
\hline 0 & 0 & 0 & $0 \times 10^{0}$ \\
0.065 & 0.06285473 & 0.0628501 & $4.650 \times 10^{-06}$ \\
0.13 & 0.12199129 & 0.1219728 & $1.850 \times 10^{-05}$ \\
0.195 & 0.17782512 & 0.1777914 & $3.370 \times 10^{-05}$ \\
0.259 & 0.23070570 & 0.2306651 & $4.060 \times 10^{-05}$ \\
0.324 & 0.28092982 & 0.2808945 & $3.530 \times 10^{-05}$ \\
0.389 & 0.32875164 & 0.3287292 & $2.250 \times 10^{-05}$ \\
0.454 & 0.37439053 & 0.3743805 & $1.000 \times 10^{-05}$ \\
0.519 & 0.41803711 & 0.4180342 & $2.920 \times 10^{-06}$ \\
0.584 & 0.45985807 & 0.4598575 & $5.980 \times 10^{-07}$ \\
0.648 & 0.5 & 0.5 & $0 \times 10^{0}$ \\
\hline
\end{tabular}

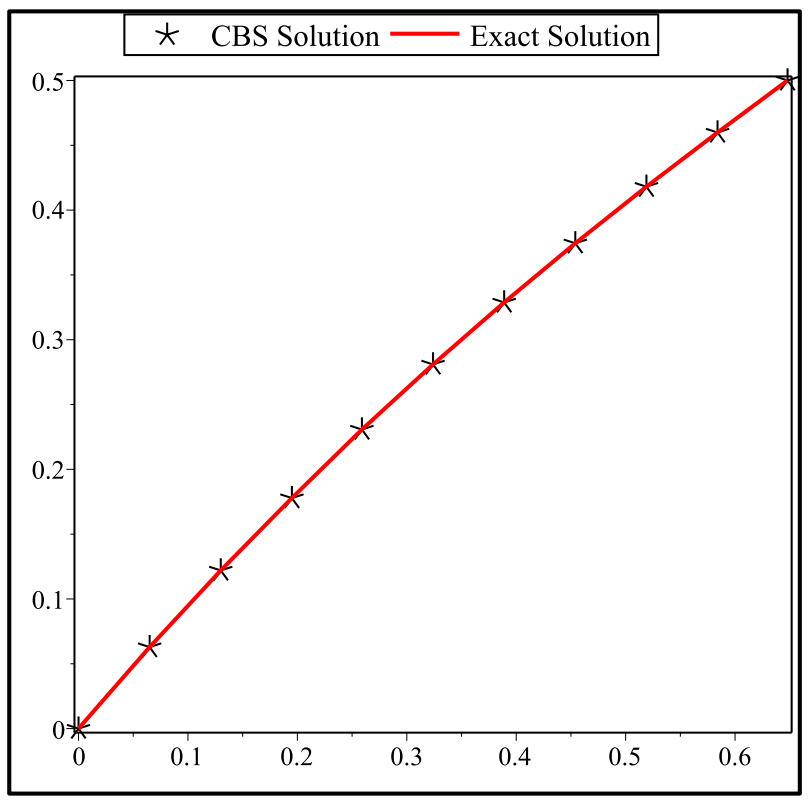

Figure 4. Problem 1 at $h=0.064872$. 


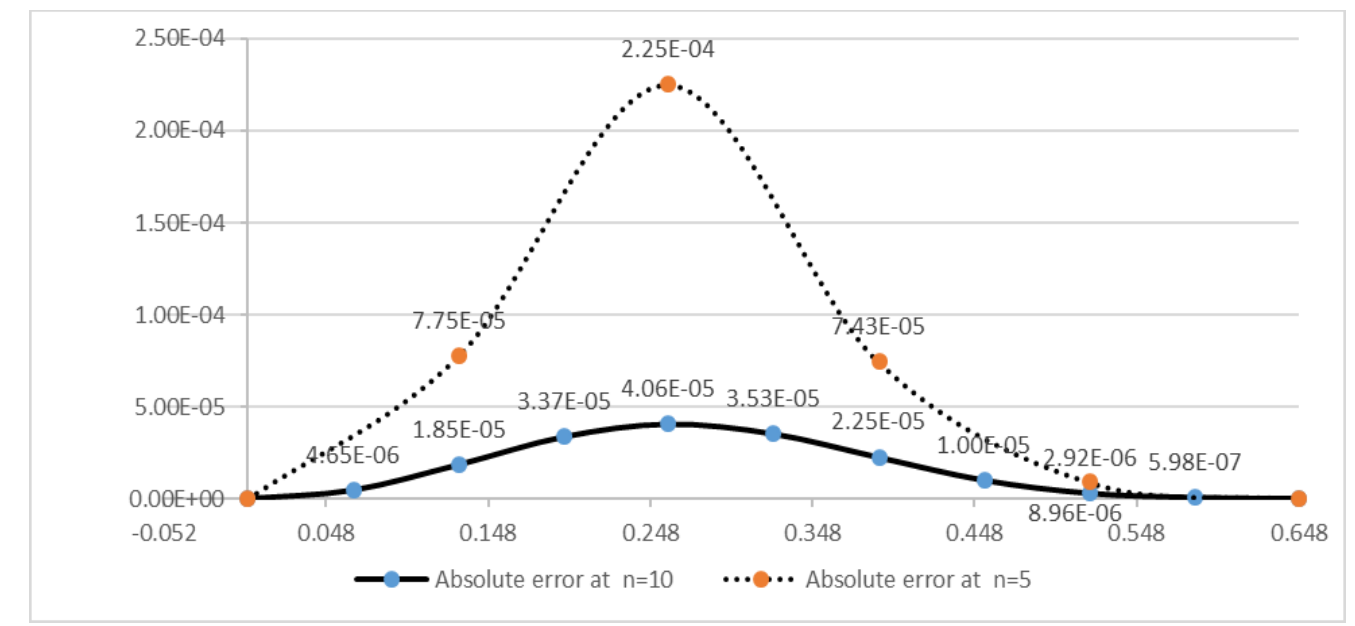

Figure 5. Problem 2 at $h=0.064872$ and $h=0.12974426$.

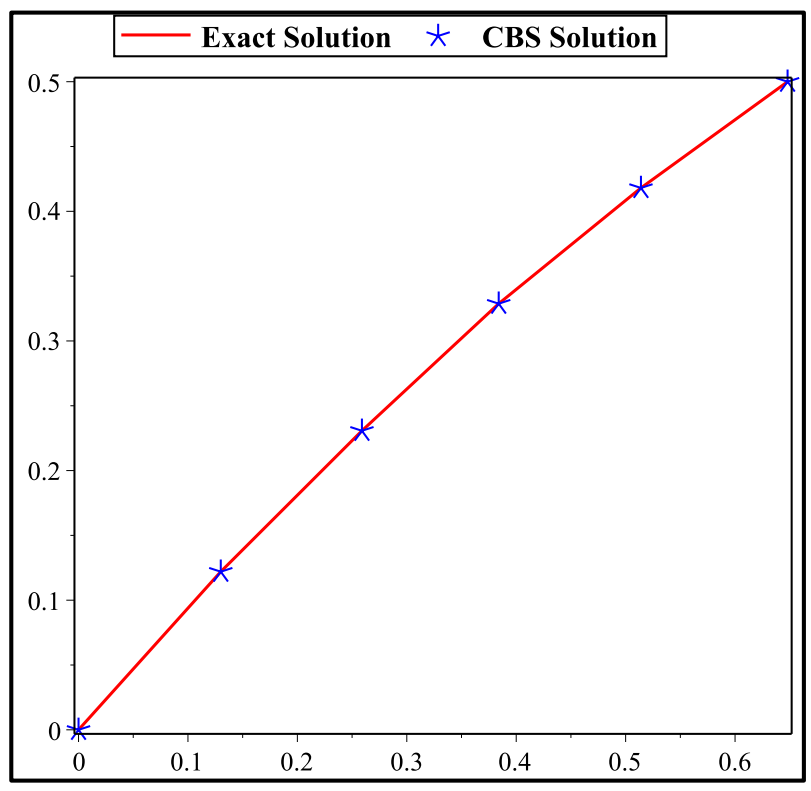

Figure 6. Problem 1 at $h=0.12974426$.

Table 6. Analyzing the exact solution and CBS solution of problem 2 at $h=0.12974426$.

\begin{tabular}{llll}
\hline $\boldsymbol{\kappa}$ & Exact Solution & CBS Solution & Absolute Error of CBS \\
\hline 0 & 0 & 0 & $0 \times 10^{0}$ \\
0.130 & 0.1219912 & 0.1219138 & $7.750 \times 10^{-05}$ \\
0.259 & 0.2307057 & 0.2304804 & $2.250 \times 10^{-04}$ \\
0.389 & 0.3287516 & 0.3286773 & $7.430 \times 10^{-05}$ \\
0.519 & 0.41803711 & 0.4180281 & $8.960 \times 10^{-06}$ \\
0.649 & 0.5 & 0.5 & $0 \times 10^{0}$ \\
\hline
\end{tabular}


Table 7. Errors at derivatives where BCs are defined in problem 2 at $h=0.064872$.

\begin{tabular}{lllll}
\hline$\kappa$ & $\begin{array}{l}\text { CBS Solution of } \\
\boldsymbol{w}^{(\mathbf{1})}(\boldsymbol{\kappa})\end{array}$ & $\begin{array}{l}\text { CBS Solution of } \\
\boldsymbol{w}^{(\mathbf{2})}(\boldsymbol{\kappa})\end{array}$ & $\begin{array}{l}\text { CBS Solution of } \\
\boldsymbol{w}^{(3)}(\boldsymbol{\kappa})\end{array}$ & $\begin{array}{l}\text { CBS Solution of } \\
\boldsymbol{w}^{(\mathbf{4})}(\boldsymbol{\kappa})\end{array}$ \\
\hline 0 & 1 & -1 & 2 & -6 \\
\hline 0.065 & 0.93893 & -0.88288 & 1.67530 & -4.66533031 \\
0.13 & 0.88490 & -0.78264 & 1.42971 & -3.68168892 \\
0.195 & 0.83690 & -0.69738 & 1.20485 & -2.94393959 \\
0.259 & 0.79396 & -0.62632 & 1.00104 & -2.38195984 \\
0.324 & 0.75524 & -0.56751 & 0.83630 & -1.94791017 \\
0.389 & 0.72004 & -0.51781 & 0.72045 & -1.60848492 \\
0.454 & 0.68786 & -0.47403 & 0.64400 & -1.34006674 \\
0.519 & 0.65840 & -0.43426 & 0.58187 & -1.12562522 \\
0.584 & 0.63139 & -0.39854 & 0.51160 & -0.95268994 \\
0.648 & 0.60653 & -0.36788 & 0.44626 & -0.81200035 \\
\hline
\end{tabular}

\subsection{Problem 3}

We consider the following equation as given in $[29,33]$

$$
w^{(10)}(\kappa)+e^{-\kappa}(w(\kappa))^{2}=e^{-3 \kappa}+e^{-\kappa} ; 0 \leq z^{\prime} \leq 1
$$

subject to BCs;

$$
\begin{gathered}
w(0)=w^{(2)}(0)=w^{(4)}(0)=-w^{(1)}(0)=-w^{(3)}(0)=1 \\
w(0)=w^{(2)}(0)=w^{(4)}(0)=-w^{(1)}(0)=-w^{(3)}(0)=e^{-1}
\end{gathered}
$$

the exact solution of given equation is $w(\kappa)=e^{-\kappa}$. The values of fifteen unknowns $j_{i}$ the Equations

$$
\begin{gathered}
(18)-(20) \text { are } \\
\jmath_{-2}=1.21938333, \\
\jmath_{-1}=1.10333333, \\
\jmath_{0}=0.99833333, \\
\jmath_{1}=-0.9033333, \\
\jmath_{2}=-0.5333053,
\end{gathered}
$$$$
\begin{aligned}
& J_{3}=-0.73961579, \\
& J_{4}=-0.66924328, \\
& J_{5}=0.605557470, \\
& J_{6}=0.547923909, \\
& J_{7}=0.495772367,
\end{aligned}
$$$$
{ }_{18}=-0.44858605 \text {, }
$$$$
1_{9}=0.405893650 \text {, }
$$$$
J_{10}=0.36726630 \text {, }
$$$$
J_{11}=0.33231776,
$$$$
J 12=0.30069852 \text {. }
$$

Tables 8 and 9 analyzed the exact solution and cubic B-spline scheme (CBS) solution of problem 3 at $h=\frac{1}{10}$ and $h=\frac{1}{5}$ respectively. Figures 7-9 analyze the exact solution with cubic B-spline scheme (CBS) solution of problem 3 at $h=\frac{1}{10}$ and $h=\frac{1}{5}$ graphically. Table 10 analyze the errors at those derivatives where boundary conditions (BCs) are defined in problem 3 at $h=\frac{1}{10}$.

Table 8. Analyzing exact solution and CBS solution of problem 3 at $h=\frac{1}{10}$.

\begin{tabular}{llll}
\hline$\kappa$ & $\begin{array}{l}\text { Exact } \\
\text { Solution }\end{array}$ & CBS Solution & $\begin{array}{l}\text { Absolute Error of } \\
\text { CBS }\end{array}$ \\
\hline 0 & 1 & 1 & 0 \\
0.1 & 0.9048374 & 0.9048417 & $4.250 \times 10^{-06}$ \\
0.2 & 0.8187308 & 0.8187471 & $1.630 \times 10^{-05}$ \\
0.3 & 0.7408182 & 0.7408483 & $3.010 \times 10^{-05}$ \\
0.4 & 0.6703200 & 0.6703577 & $3.770 \times 10^{-05}$ \\
0.5 & 0.6065307 & 0.6065662 & $3.550 \times 10^{-05}$ \\
0.6 & 0.5488116 & 0.5488376 & $2.590 \times 10^{-05}$ \\
0.7 & 0.4965853 & 0.4965999 & $1.460 \times 10^{-05}$ \\
0.8 & 0.4493290 & 0.4493350 & $6.080 \times 10^{-06}$ \\
0.9 & 0.4065697 & 0.4065712 & $1.500 \times 10^{-06}$ \\
1 & 0.3678794 & 0.3678794 & 0 \\
\hline
\end{tabular}




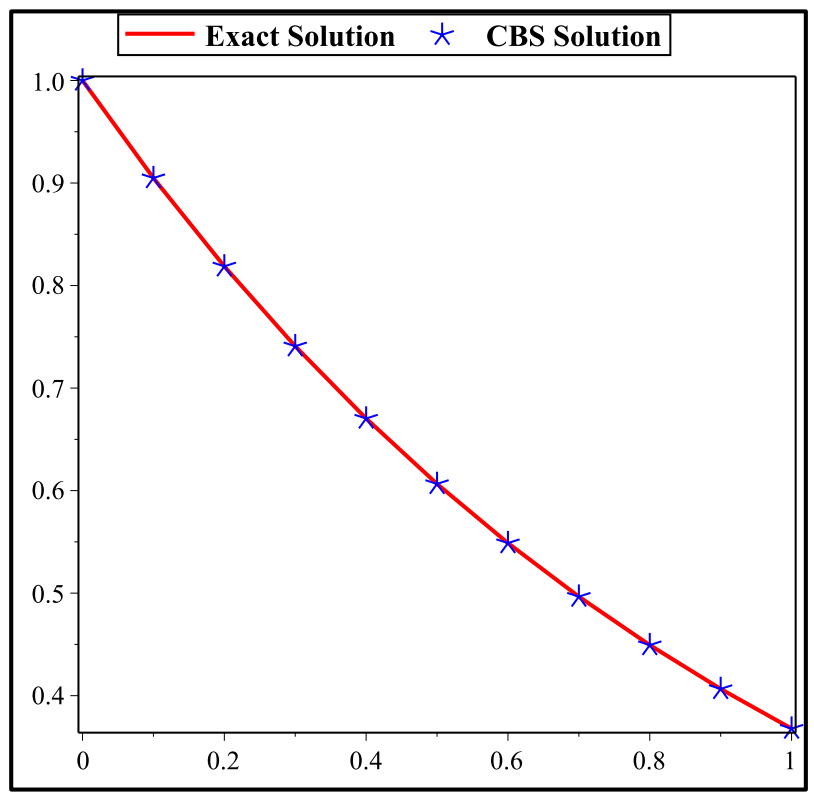

Figure 7. Problem 1 at $h=\frac{1}{10}$.

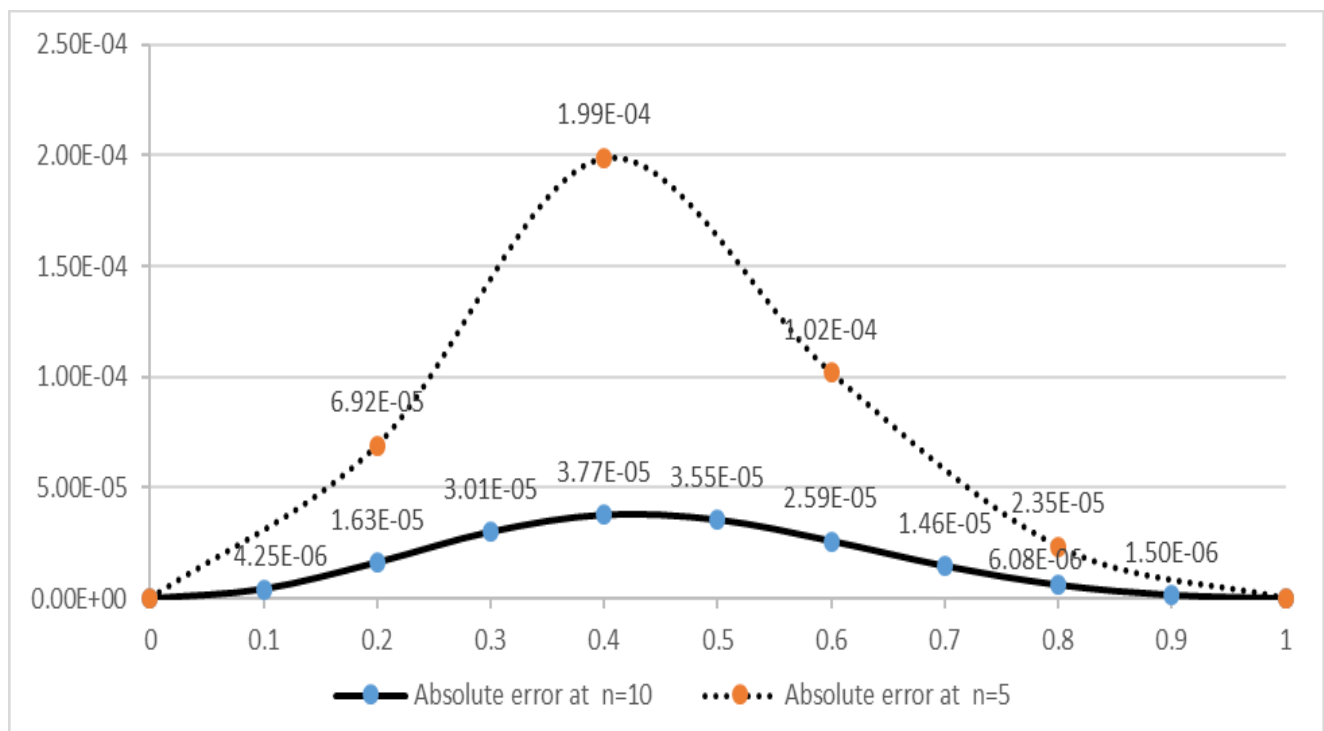

Figure 8. Problem 3 at $h=\frac{1}{10}$ and $h=\frac{1}{5}$.

Table 9. Analyzing exact solution and CBS solution of problem 3 at $h=\frac{1}{5}$.

\begin{tabular}{llll}
\hline$\kappa$ & Exact Solution & CBS & Absolute Error of CBS \\
\hline 0 & 1 & 1 & $0 \times 10^{0}$ \\
0.2 & 0.8187308 & 0.8188000 & $6.920 \times 10^{-05}$ \\
0.4 & 0.6703200 & 0.6705188 & $1.990 \times 10^{-04}$ \\
0.6 & 0.5488116 & 0.5489132 & $1.020 \times 10^{-04}$ \\
0.8 & 0.4493290 & 0.4493525 & $2.350 \times 10^{-05}$ \\
1 & 0.3678794 & 0.3678794 & $0 \times 10^{0}$ \\
\hline
\end{tabular}


Table 10. Errors at derivatives where BCs are defined in problem 3 at $h=\frac{1}{10}$.

\begin{tabular}{lll}
\hline$\kappa$ & CBS Solution of $w^{(2)}(\kappa), w^{(4)}(\kappa)$ & CBS Solution of $w^{(1)}(\kappa), w^{(3)}(\kappa)$ \\
\hline 0 & 1 & -1 \\
0.1 & 0.90482409 & -0.90484731 \\
0.2 & 0.81870008 & -0.81872330 \\
0.3 & 0.74077662 & -0.74079984 \\
0.4 & 0.67027318 & -0.67029640 \\
0.5 & 0.60648354 & -0.60650676 \\
0.6 & 0.54876876 & -0.54879198 \\
0.7 & 0.49655070 & -0.49657392 \\
0.8 & 0.44930633 & -0.44932955 \\
0.9 & 0.40656241 & -0.40658563 \\
1 & 0.36787944 & -0.36787944 \\
\hline
\end{tabular}

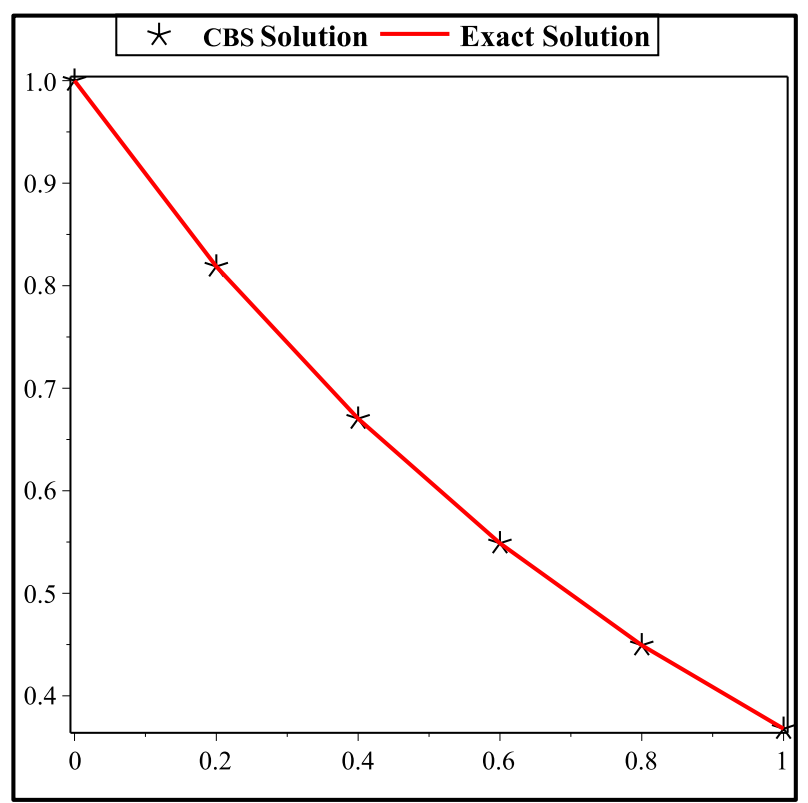

Figure 9. Problem 1 at $h=\frac{1}{10}$ and $h=\frac{1}{5}$.

\section{Conclusions}

In this study, we present new scheme using CBS of some non-linear differential equations arising in visco-elastic flows and hydrodynamic stability problems. The proper selection for the choice of the scheme and an appropriate of adjustment BCs may cause elasticity for the betterment of the results. The new CBS scheme proposed in this study is very simple to apply in solving the non-linear DEs compared with some existing schemes. An advantage of using the CBS scheme is that it gives a spline function on each new time line which can be applied to achieve the numerical solutions at any stage in the space direction.

Author Contributions: Conceptualization, A.T., A.G. and A.K.; methodology, A.T., A.K. and M.N.N.; software, A.T., A.K., F.K. and K.S.N; formal analysis, S.A.A.K., K.S.N. and F.K.; writing-original draft preparation, A.K., A.G. and S.A.A.K.; writing-review and editing, A.T., K.S.N. and A.G.; visualization, F.K., M.N.N. and A.G.; supervision, M.N.N. and S.A.A.K.; funding acquisition, A.T.

Funding: This research is funded by Deanship of Scientific Research at Majmaah University, Project Number (R-1441-25).

Acknowledgments: The first author Asifa Tassaddiq (A.T) would like to thank Deanship of Scientific Research at Majmaah University, for supporting this work under Project Number (R-1441-25).

Conflicts of Interest: The authors declare no conflict of interest. 


\section{References}

1. Agarwal, R.P. Boundary Value Problems from Higher Order Differential Equations; World Scientific Singapore: Singapore, 1986.

2. Al-Hayani, W. Adomian decomposition method with green's function for solving tenth-order boundary value problems. Appl. Math. 2014, 5, 1437. [CrossRef]

3. Al-Hayani, W. Adomian decomposition method with green's function for solving twelfth-order boundary value problems. Appl. Math. Sci. 2015, 9, 353-368. [CrossRef]

4. Barari, A.; Omidvar, M.; Najafi, T.; Ghotbi, A.R. Homotopy perturbation method for solving tenth order boundary value problems. Int. J. Math. Comput. 2009, 3, 15-27.

5. Chandrasekhar, S. Hydrodynamic and Hydromagnetic Stability; Oxford University Press: London, UK, 1961.

6. Burns, W.W.; Owen, H.A.; Wilson, T.G.; Rodriguez, G.E.; Paulkovich, J. A digital computer simulation and study of a direct-energy-transfer power-conditioning system. In Proceedings of the IEEE Power Electronics Specialists Conference, Culver City, CA, USA, 9-11 June 1975; pp. 138-149.

7. Djidjeli, K.; Twizell, E.; Boutayeb, A. Numerical methods for special nonlinear boundary-value problems of order 2 m. J. Comput. Appl. Math. 1993, 47, 35-45. [CrossRef]

8. Erturk, V.S.; Momani, S. A reliable algorithm for solving tenth-order boundary value problems. Numer. Algorithms 2007, 44, 147-158. [CrossRef]

9. Mohyud-Din, S.T.; Yildirim, A. Solution of tenth and ninth-order boundary value problems by homotopy perturbation method. J. Korean Soc. Ind. Appl. Math. 2010, 14, 17-27.

10. Mohyud-Din, S.T.; Yildirim, A. Solutions of tenth and ninth-order boundary value problems by modified variational iteration method. Appl. Appl. Math. 2010, 5, 11-25.

11. Noor, M.A.; Al-Said, E.; Mohyud-Din, S.T. A reliable algorithm for solving tenth-order boundary value problems. Appl. Math. 2012, 6, 103-107.

12. Wazwaz, A.-M. Approximate solutions to boundary value problems of higher order by the modified decomposition method. Comput. Math. Appl. 2000, 40, 679-691. [CrossRef]

13. Wazwaz, A.-M. The modified adomian decomposition method for solving linear and nonlinear boundary value problems of tenth-order and twelfth-order. Int. J. Nonlinear Sci. Numer. Simul. 2000, 1, 17-24. [CrossRef]

14. Wazwaz, A.-M. The numerical solution of special eighth-order boundary value problems by the modified decomposition method. Neural Parallel Sci. Comput. 2000, 8, 133-146.

15. Wazwaz, A.-M. The numerical solution of sixth-order boundary value problems by the modified decomposition method. Appl. Math. Comput. 2001, 118, 311-325. [CrossRef]

16. Taiwo, O.A.; Ogunlaran, O.M. A non-polynomial spline method for solving linear fourth-order boundary-value problems. Int. J. Phys. Sci. 2011, 13, 3246-3254.

17. Hamdy, A.; Badreddin, E. Dynamic modeling of a wheeled mobile robot for identification, navigation and control. In IMACS Conference on Modeling and Control of Technological Systems; IMAS: Lille, France, 1992; pp. 119-128.

18. Hughes, J.T. Type-C Wind Turbine Model Order Reduction and Parameter Identification. Master's Thesis, University of Illinois at Urbana-Champaign, Champaign, IL, USA, 2013.

19. Kanwal, G.; Ghaffar, A.; Hafeezullah, M.M.; Manan, S.A.; Rizwan, M.; Rahman, G. Numerical solution of 2-point boundary value problem by subdivision scheme. Commun. Math. Appl. 2019, 10,1-11.

20. Manan, S.A.; Ghaffar, A.; Rizwan, M.; Rahman, G.; Kanwal, G. A subdivision approach to the approximate solution of 3rd order boundary value problem. Commun. Math. Appl. 2018, 9, 499-512.

21. Gupta, Y.; Kumar, M. B-spline method for solution of linear fourth order boundary value problem. System 2011, 1, 4 .

22. Islam, M.S.; Hossain, M.B.; Rahman, M.A. Numerical approaches for tenth and twelfth order linear and nonlinear differential equations. Br. J. Math. Comput. Sci. 2015, 5, 637. [CrossRef]

23. Khalid, A.; Naeem, M.N. Cubic B-spline solution of nonlinear sixth order boundary value problems. J. Math. 2018, 50, 91-103.

24. Khalid, A.; Naeem, M.N.; Ullah, Z.; Ghaffar, A.; Baleanu, D.; Nisar, K.S.; Al-Qurashi, M.M. Numerical solution of the boundary value problems arising in magnetic fields and cylindrical shells. Mathematics 2019, 7, 508. [CrossRef] 
25. Lang, F.-G.; Xu, X.-P. A new CBS method for linear fifth order boundary value problems. J. Appl. Math. Comput. 2011, 36, 101-116. [CrossRef]

26. Siddiqi, S.S.; Akram, G. Solution of 10th-order boundary value problems using non-polynomial spline technique. Appl. Math. Comput. 2007, 190, 641-651. [CrossRef]

27. Siddiqi, S.S.; Akram, G. Solutions of tenth-order boundary value problems using eleventh degree spline. Appl. Math. Comput. 2007, 185, 115-127. [CrossRef]

28. Twizell, E.H.; Boutayeb, A.; Djidjeli, K. Numerical methods for eighth-, tenth-and twelfth-order eigenvalue problems arising in thermal instability. Adv. Comput. Math. 1994, 2, 407-436. [CrossRef]

29. Kasi Viswanadham, K.N.S.; Ballem, S. Numerical solution of tenth order boundary value problems by galerkin method with quintic B-splines. Int. J. Innov. Sci. Math. 2014, 2, 288-294.

30. Kasi Viswanadham, K.N.S.; Ballem, S. Numerical solution of tenth order boundary value problems by galerkin method with septic B-spline. Int. J. Appl. Sci. Eng. 2015, 13, 247-260.

31. Kasi Viswanadham, K.N.S.; Raju, Y.S. Quintic B-spline collocation method for tenth order boundary value problems. Bound. Value Probl. 2012, 51, 7-13.

32. Kasi Viswanadham, K.N.S.; Raju, Y.S. Sextic B-spline collocation method for eighth order boundary value problems. Int. J. Appl. Sci. Eng. 2014, 12, 43-57.

33. Kasi Viswanadham, K.N.S.; Ballem, S. Numerical solution of eighth order boundary value problems by galerkin method with septic B-splines. Procedia Eng. 2015, 127, 1370-1377.

(c) 2019 by the authors. Licensee MDPI, Basel, Switzerland. This article is an open access article distributed under the terms and conditions of the Creative Commons Attribution (CC BY) license (http://creativecommons.org/licenses/by/4.0/). 